\title{
Efficiency Assessment of Nanosecond Laser Robotic Maxillofacial Area Surgery in Experiment
}

\author{
DOI: $10.17691 / \mathrm{stm} 2017.9 .4 .15$ \\ Received April 18, 2017
}

A.A. Chunikhin, MD, PhD, Associate Professor, Department of Oral Surgery';

Yu.V. Poduraev, DSc, Professor, Head of the Department of Robotics and Mechatronics;;

A.A. Vorotnikov, Junior Researcher, Laboratory of Industrial Robotics, Mobile and Special Robotics, Mechatronic Modules and Digital Drives;

D.D. Klimov, Junior Researcher, Laboratory of Industrial Robotics, Mobile and Special Robotics, Mechatronic Modules and Digital Drives;

M.Y. Sahakyan, MD, PhD, Associate Professor, Department of Orthopedic Stomatology and Orthodontics; Chief Doctor of the Dental Implantology Center ${ }^{3}$;

E.A. Bazikyan, MD, DSc, Professor, Head of the Department of Oral Surgery ${ }^{1}$

${ }^{1}$ Moscow State University of Medicine and Dentistry named after A.I. Evdokimov, 20/1 Delegatskaya St., Moscow, 127473, Russian Federation;

${ }^{2}$ Stankin Moscow State Technological University, Vadkovsky per., 1, Moscow, 127994, Russian Federation;

${ }^{3}$ Nizhny Novgorod State Medical Academy, 10/1 Minin and Pozharsky Square, Nizhny Novgorod, 603005,

Russian Federation

The aim of the study was to study the root mean square deviation from a given trajectory while performing standard surgical incisions using laser radiation with a tip built into the manipulating robot relative to a surgeon's arm using a manual training system.

Materials and Methods. We used a nanosecond laser device with unique radiation characteristics and a manipulating robot, which is a hinged seven-link mechanism with a sequential kinematic structure conjugated by a prototype of the working element holding the holder of the optical fiber of the medical laser on the flange of the 6-degree manipulator.

The coordinates of points were measured by typical trajectories (linear, curve, festoon) taking into account functional movements of the medical instrument in manual procedures and when being moved by the robot using a laser coordinate measuring device with its reflector being fixed on the prototype of the working element.

Results. The root mean square deviation in manual movements in a linear trajectory was found to be 11 times greater $(p \leqslant 0.05)$ than when being moved by the robot, and 5 times greater $(p \leqslant 0.05)$ in a curve trajectory.

The mean value of all root mean square deviations for each of the small circles on the festoon trajectory for manual movements was threefold $(p \leqslant 0.05)$ to when being moved by the robot. The standard deviation from the straight line equidistant from all the centers of small circles in manual movements was fourfold $(p \leqslant 0.05)$ compared to that in robot movements.

The mean value of root mean square deviation in various trajectories (linear, curve, festoon) with manual movement was 3.3 times greater than when being moved by the robot.

Conclusion. The results of the study suggest that the use of robotic laser systems in medicine, in particular, the one developed by us for the maxillofacial region, makes it possible to significantly improve the accuracy of medical laser movements, which is of prime importance in complex trajectories.

Key words: robotic surgery; nanosecond pulsed laser; photodynamic effect.

Contemporary surgery is developing along the way of modernization of minimally invasive procedures. This tendency is associated with the development of robotic and laser technologies. It is supported by breakthroughs in the design of novel devices using artificial intelligence and lasers with unique parameters [1, 2].

For contacts: Andrey A. Chunikhin, e-mail: docca74@yandex.ru 
The success rate of surgeries using robotic surgical system daVinci has led to a large-scale implementation of robotic technologies in various medical fields. The experience of using the system demonstrates significant advantages of robotic technologies over open, as well as laparoscopic surgeries [3, 4].

Currently, robotic systems are under development in the world medical industry to perform operative interventions, primarily, in the fields of angiology, gynecology, ophthalmology, among others. However, no robots have been invented yet to perform minimally invasive surgeries in head and neck areas. Operative interventions in this area require a delicate technique, since the damage of ultrafine anatomical structures, such as muscles, vessels and nerves, paranasal sinuses, can result in major complications and prolong treatment period [5].

Development and design of Russian robotic surgical systems for treating complicated maxillofacial pathologies and suitable for integrating a laser module into robot surgeon manipulators that deliver laser radiation flow in deep head and neck anatomical structures is a promising direction for medical industry aiming to implement the improvement of medical care program for Russian population.

The multifunctional robotic surgical system was developed in two stages. At first, we developed a laser module with unique characteristics, which is able to combine low- and high-intensity radiation and a photochemical effect on oxygen in tissues [6].

Then, we designed a stand for maxillofacial surgery based on a manipulating robot developed by researchers from Moscow State University of Medicine and Dentistry named after A.I. Evdokimov and Stankin Moscow State Technological University.

The aim of the study was to study the root mean square deviation from a given trajectory while performing standard surgical incisions using laser radiation with a tip built into the manipulation robot relative to a surgeon's arm using a manual training system.

Materials and Methods. The study was carried out using a robotic stand of a multifunctional surgical system, which includes the following subsystems: a manipulating robot, a manual training system, a patient's phantom head, and the surgeon's working place.

The manipulating robot (Figure 1) is a hinged sevenlink mechanism with a sequential kinematic structure. Manipulator arms have modular configuration and consist of an electric motor, a harmonic drill, a positioning transducer and a torque transducer.

The manipulating robot enables one to perform functional movements with a medical instrument, and partially performs surgeon's functions, thus enhancing surgical capabilities.

The medical instrument in the manipulating robot is a laser working element EMPD-1260-2P developed by a research group from the Oral Surgery Department, Moscow State University of Medicine and Dentistry

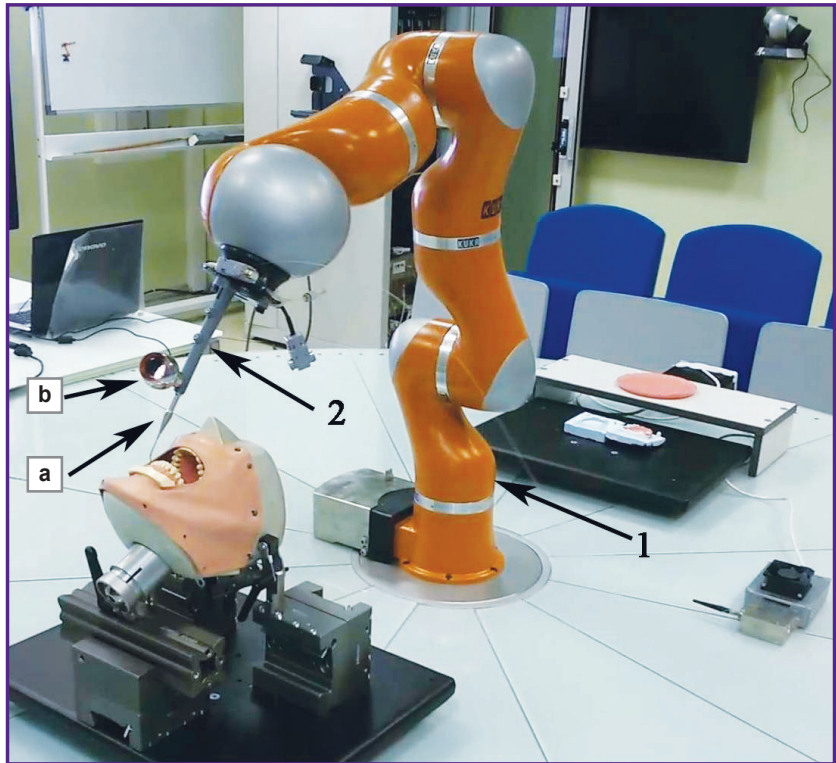

Figure 1. Manipulating robot for maxillofacial surgery: 1 - manipulator Leichtbauroboter 4+ (KUKA AG, Germany); 2 - a working organ prototype: $2 a-$ laser light guide holder; $2 b$ - laser reflector of the coordinate measuring device

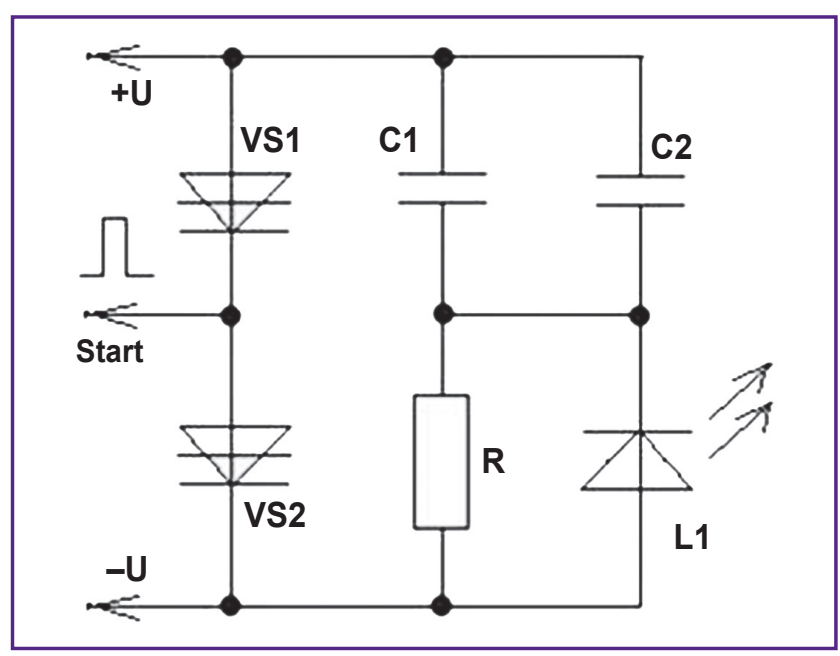

Figure 2. Principle diagram of a laser pumping driver

named after A.I. Evdokimov, and designed by LLC "New Surgical Technologies" (Moscow). Optic radiation is produced by a multimode semiconductor laser based on AlGaAs with direct current pumping.

The working element EMPD-1260-2P is a pulse pumping driver, structurally integrated with a diode-laser module, with wavelength of radiation of $\lambda=1,260 \mathrm{~nm}$.

The laser pumping driver has pulse mode that allows it to generate different optical output power due to the change in pumping current pulse frequency (Figure 2).

The pumping current driver is a discharge circuit consisting of a storage container $\mathrm{C} 1+\mathrm{C} 2$, a semiconductor laser L1, and a pair of dinisters (VS1 and VS2). The dinisters are connected in series and 
are selected according to electric parameters. A positive impulse is given on VS2 by the amplitude, which exceeds turn-on voltage. After VS2 opening, all power supply voltage passes to VS1, which opens as well. Total turn-on voltage of this pair of dinisters exceeds the laser power supply $U$ by a few volts only. A circuit capacitance discharge goes through open VS1 and VS2, as well as through the laser (L1). This is how a pumping current impulse is formed. Capacitors C1 and C2 are being charged during the pause between the initiating pulses through a charging resistor $\mathrm{R}$ simultaneously switched with the laser L1.

An output optic cable of the laser is a light guide with a quartz core, $600 \mu \mathrm{m}$ in diameter, covered by a protective plastic shell. The optic cable is radiotransparent, it is capable of transmitting radiation with the wavelength up to $2.5 \mu \mathrm{m}$. Minimal bending radius of the cable is $40 \mathrm{~mm}$, by core strength condition. Its maximal length is $3 \mathrm{~m}$. The transmission coefficient of the optic cable is not less than $80 \%$. The light guide numerical aperture is $f / 0.22$. Laser radiation affects biological tissues directly from a distal light guide end.

To control laser behavior mounted on a multi-stage robot, it is necessary to connect up a power supply via an electromagnetic relay to digital output of the robot control unit.

In the manipulator controller there is a 16-channel modulator of discrete output KL2809 (Beckhoff Automation, Germany).

To switch laser power unit channels in response to a control signal from the robot controller, an electromagnetic relay PE034024 (TE Connectivity, Switzerland) is used. The relay controls the signal $+24 B$ supplied by a discrete output block KL2809.

To measure the coordinate points in functional movements and typical trajectories, we used a laser coordinate measuring device Leica Laser Tracker LTD800 (Leica Geosystems, Switzerland).

To measure the coordinates of the points, we used a portable photogrammetric coordinate measuring device Actiris 350 (ActiCM, France).

We developed a prototype of the working element in the subsystem of the manual training system designed to determine the position and attitude of the medical instrument when it is manually moved, and to perform training functions of the robot stand (Figure 3). The working element prototype is designed to join an optic light guide holder of the medical laser with the flange of the 6-degree manipulator and to fix a laser reflector of the coordinate measuring machine on it, which is required to control the position and speed of the working element in its movement along the trajectory.

The designed model of the working element was printed using a 3D printer WANHAO Duplicator 6 (WANHAO Precision Casting Co., Ltd, China) made of heat-resistant plastic.

Experimental investigations were carried out on standard trajectories (Figure 4) accounting for functional

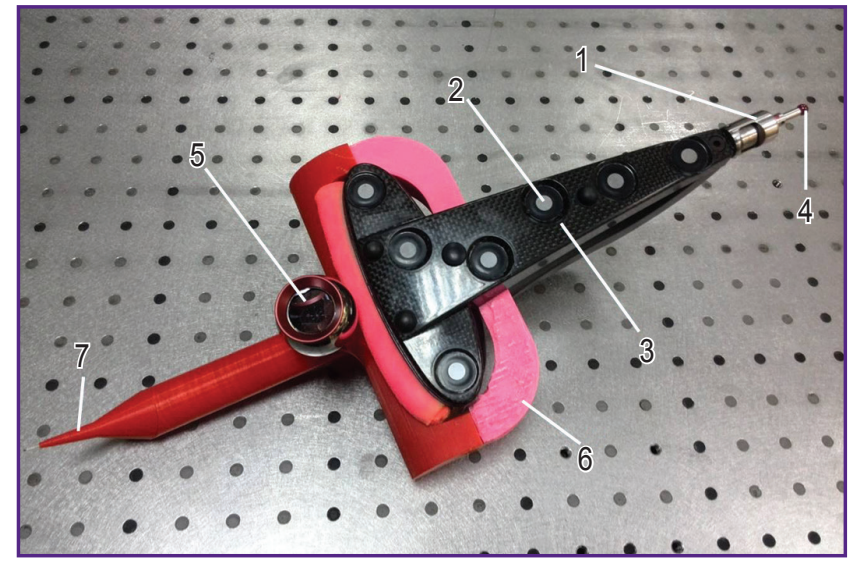

Figure 3. A working element prototype of the manual training complex:

1 - probe of the photogrammetric coordinate measuring device; 2 - reflector; 3 - rigid base; 4 - ball ruby attachment; 5 - angle reflector of the laser coordinate measuring system; 6 - bonding tool; 7 - cap imitating a medical instrument

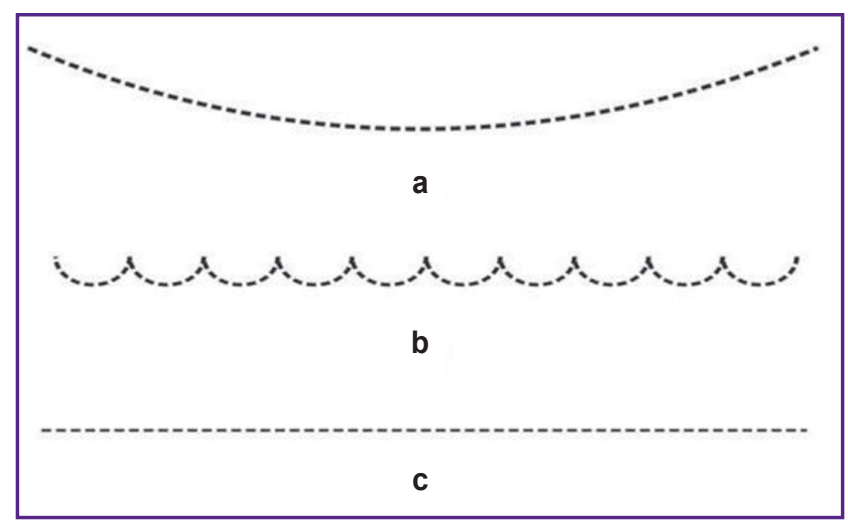

Figure 4. Standard functional trajectories of a medical laser:

(a) curve; (b) festoon; (c) linear

movements of the medical instrument while performing surgical procedures using a laser instrument.

To measure the coordinates of the points on standard trajectories, a laser tracker reflector was mounted on the medical instrument. The coordinates were measured by scanning with sampling frequency of at least $200 \mathrm{~Hz}$.

Root mean square deviation value in different measurable values was calculated as follows:

$$
\sigma=\sqrt{\frac{1}{n} \cdot \sum_{i=1}^{n}\left(p_{i}-p_{m}\right)^{2}}
$$

where $p_{m}$ is a mean value of the measurable value; $p_{i}$ is the measurable value of the variable at each point $i$; $n$ is the number of points. The experiment was repeated 10 times with the subsequent calculation of an average value to obtain statistically significant results.

Results and Discussion. After performing measurements, we calculated root mean square deviations from linear $\sigma_{l}$, curve $\sigma_{c}$ and festoon $\sigma_{f}$ 


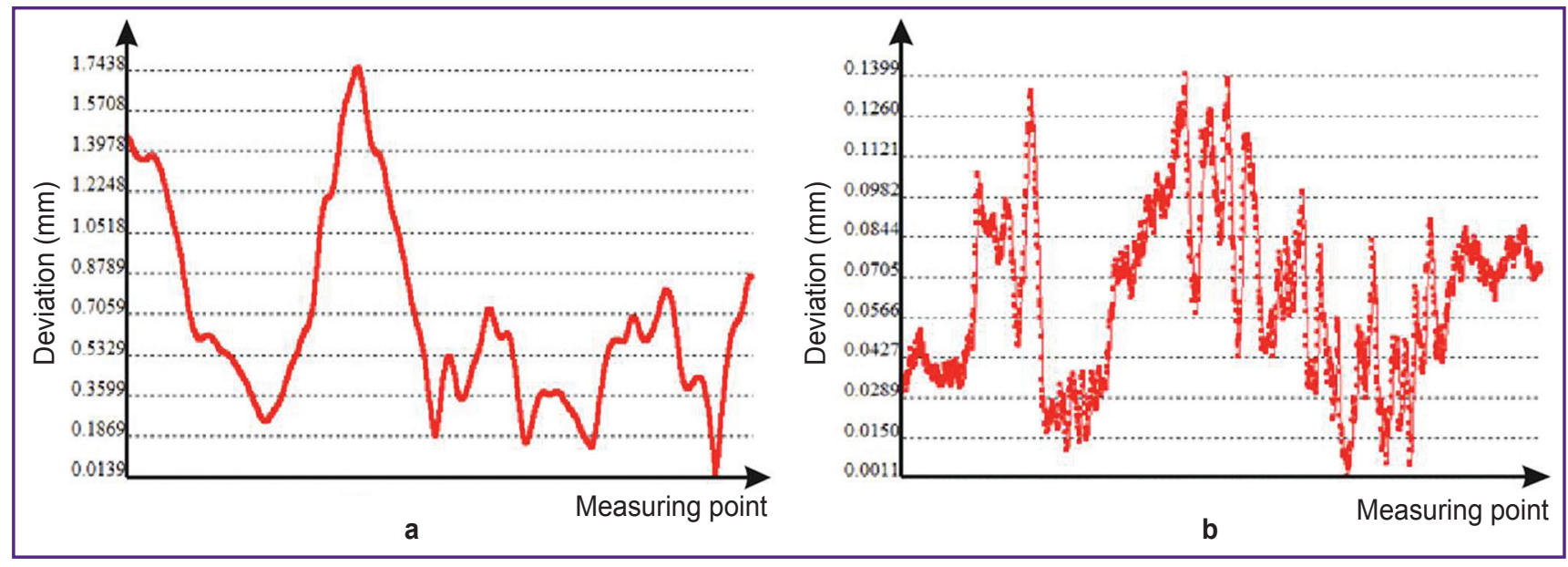

Figure 5. Deviations of coordinates from the straight line along a linear trajectory:

(a) a trajectory drawn by a human; (b) a robot-made path
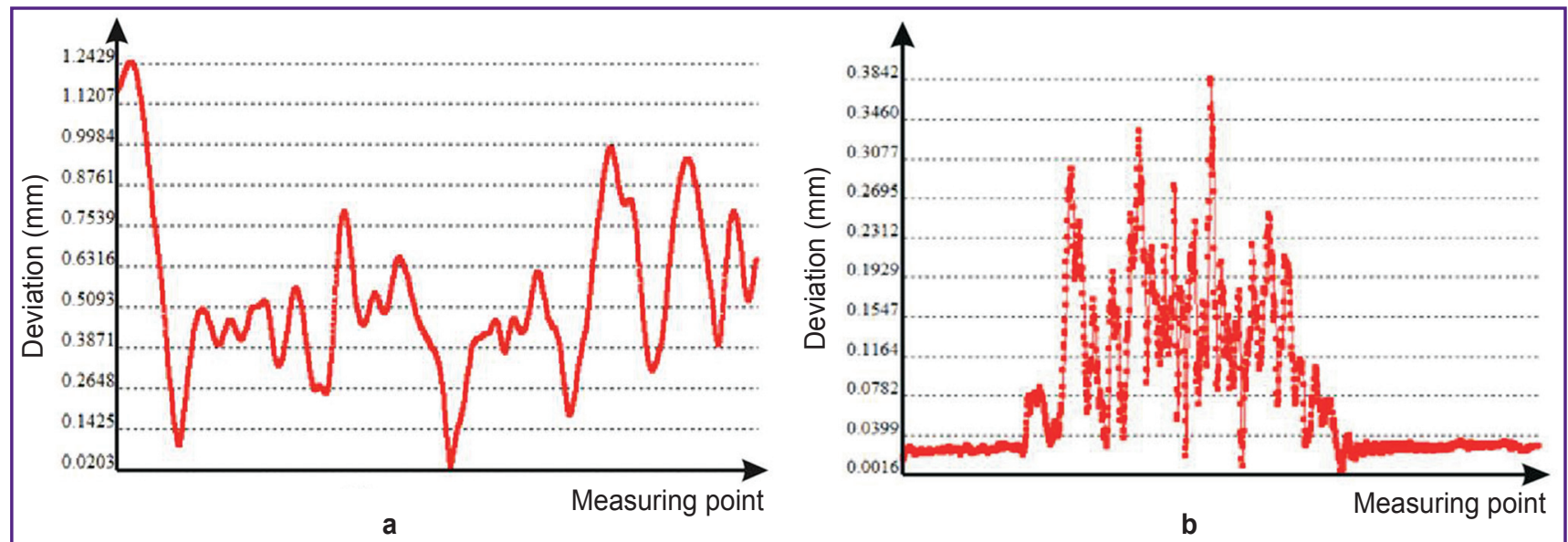

Figure 6. Deviations of coordinate points from the circle in a curve trajectory:

(a) a trajectory drawn by a human; (b) a robot-made path

trajectories. A root mean square deviation of points from the trajectory is the deviation value of every measured trajectory point from its projection to the midline (model) drawn according to the least square method (mean square deviation of points from a trajectory in manual movements has $\mathrm{H}$ index, when moved by a robot $\mathrm{R}$ index).

In the case of a linear trajectory, the midline is a straight line. Figure 5 (a) represents a deviation graph of the line of the measured coordinates during manual movements, and Figure 5 (b) shows those during robot movements.

According to the above formula we calculated mean square deviation values of the measured coordinates from the straight line, when moved by a robot $\sigma_{R l}=0.035 \mathrm{~mm}$, and in manual movements $\sigma_{H}=0.393 \mathrm{~mm}$.

In a curve trajectory the midline is the arc of a circle. Figure 6 represents the graph of the deviations of the measured points in manual movements (a), and in robot movements (b).
According to the above mentioned formula we determined the values of mean square deviations of the measured coordinate points from the circle when moved by a robot $\sigma_{R l}=0.054 \mathrm{~mm}$, and in manual movements $\sigma_{H I}=0.294 \mathrm{~mm}$.

In contrast to linear and curve trajectories, a festoon one is complex. We considered it as a sum of simple paths: one linear and several curve trajectories. Thus, a festoon trajectory includes several minor circles (the current study contains eight), the centers of which are in line with one another. A set of scanned points (purple and bright green) of medical instrument motion path, and their midlines in manual movements are given in Figure 7 (a), in robot motion - in Figure 7 (b).

The procedure of determining root mean square deviations from festoon trajectory coordinates included several steps.

1. Determination of root mean square deviations from each minor circle according to the above mentioned formula. The Table shows the values of the deviations in 


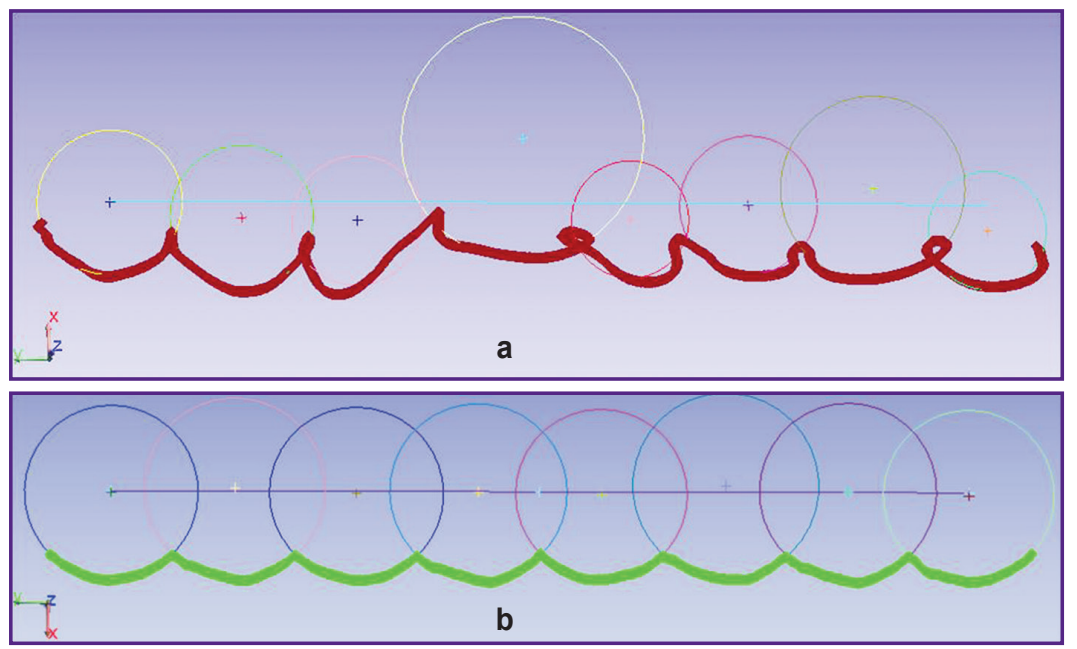

Figure 7. A set of scanned points of a medical instrument motion trajectory along a festoon trajectory:

(a) a trajectory drawn by a human; (b) a robot-made path

Mean root square deviations from each of minor circles of the measured coordinate points of a festoon trajectory, and the diameters of the trajectories $(\mathrm{mm})$

\begin{tabular}{ccccccccc}
\hline \multirow{2}{*}{ Parameters } & \multicolumn{7}{c}{ Circle number } \\
& $\mathbf{1}$ & $\mathbf{2}$ & $\mathbf{3}$ & $\mathbf{4}$ & $\mathbf{5}$ & $\mathbf{6}$ & $\mathbf{7}$ & $\mathbf{8}$ \\
\hline$\sigma_{\text {Hic }}$ & 0.129 & 0.077 & 0.34 & 0.181 & 0.245 & 0.148 & 0.123 & 0.163 \\
\hline$\sigma_{R i c}$ & 0.05 & 0.067 & 0.066 & 0.03 & 0.065 & 0.035 & 0.065 & 0.048 \\
\hline$D_{H}$ & 9.544 & 9.456 & 8.582 & 15.984 & 7.692 & 9.09 & 12.124 & 7.77 \\
\hline$D_{R}$ & 13.976 & 14.48 & 15.214 & 14.052 & 14.508 & 14.24 & 14.814 & 14.208 \\
\hline
\end{tabular}

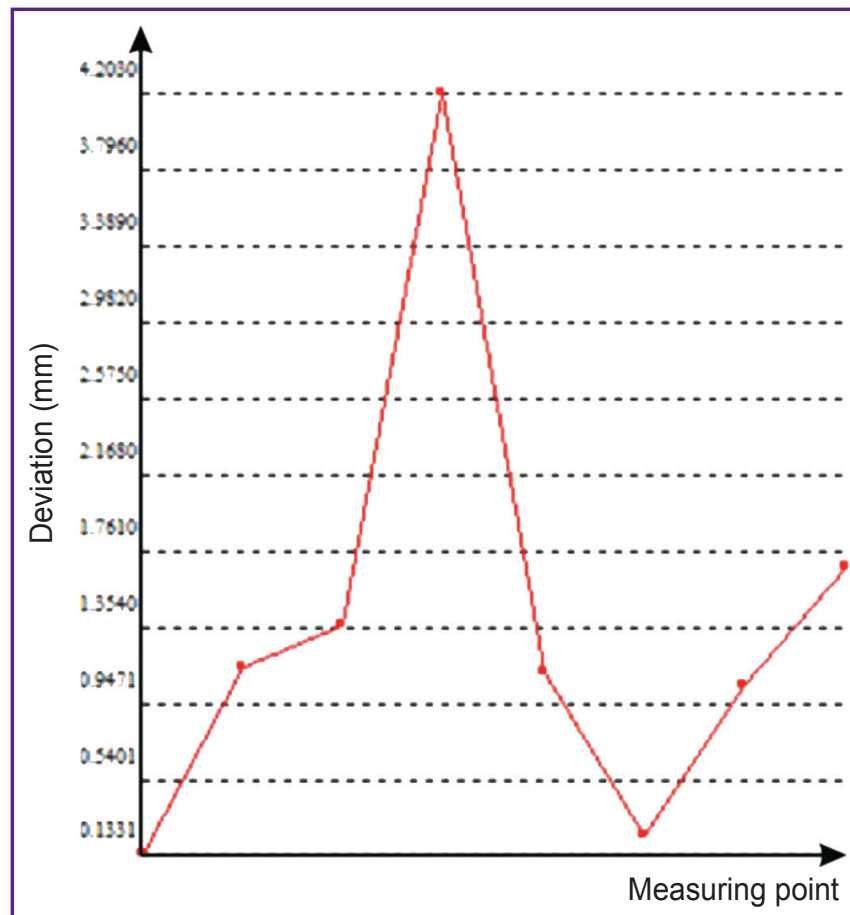

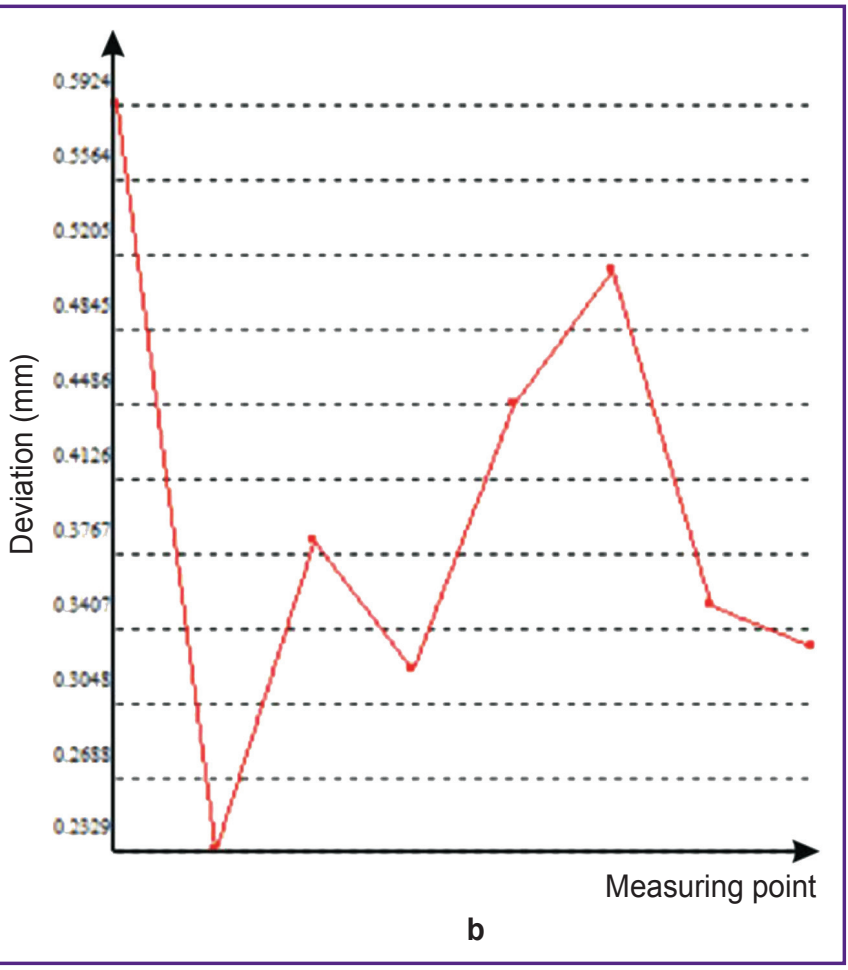

Figure 8. Deviations of the coordinates of the centers of small circles from the straight line along a festoon trajectory: (a) a trajectory drawn by a human; (b) a robot-made path 
measured coordinates for manual movements and those of a robot. There is the spread in $\sigma_{H F C}$ values in relation to $\sigma_{R f c}$ on each circumference.

2. Determination of the mean value of all root mean square deviations in each of minor circles on a festoon trajectory. For manual movements $\sigma_{\text {Hfcm }}=0.176 \mathrm{~mm}$, in robot motion $\sigma_{\text {Rfom }}=0.053 \mathrm{~mm}$.

3. Determination of the root mean square deviation from the equidistance line of all the obtained centers of minor circles (Figure 8). It is necessary because when trajectory points were generated, the centers of all circles were on one and same line in movements carried out by a human as well as those by a robot. A root mean square deviation value in accordance with the mentioned formula was calculated for robot movements as $\sigma_{R f}=0.206 \mathrm{~mm}$, and for manual movements as $\sigma_{H f}=0.902 \mathrm{~mm}$. In manual movements (Figure 8 (a)) there is a significant curve, which has an effect on $\sigma_{H f f}$. Due to the fact that in manual movements, accidental errors can prevail, we made a decision not to ignore this curve.

It should be noted that when determining the diameters of each minor circle by the measured points of a festoon trajectory in both: manual $\left(D_{H}\right)$, and automatic $\left(D_{R}\right)$ movements (see the Table) we revealed a significant error in relation to the specified size. The error can be of different nature: in case of manual movements it relates to a human factor, and in case of automatic movements - to non-rigidity of the robot, or an error in processing the algorithm of experimental findings related to a small arc of the circle (a laser tracker software Spatial Analyzer, New River Kinematics, USA).

The data processing showed that the root mean square deviation of the points from a linear trajectory is 11 times greater $(p \leq 0.05)$ in manual movement than in robot movements. The root mean square deviation of the points from a curve trajectory is 5 times greater $(p \leq 0.05)$ in manual movements than in robot movements.

A festoon trajectory was assessed using two components: a root mean square deviation in each minor circle, and the straight line equidistant from all the centers of small circles. The mean value of all deviations in each of small circles on a festoon trajectory in manual movements was threefold $(p \leq 0.05)$ compared to that in robot movements. The root mean square deviation from the straight line equidistant from all the centers of small circles in manual movements was fourfold $(p \leq 0.05)$ compared to robot movements.

Thus, root mean square deviation from different trajectories (linear, curve, festoon) in manual movements is 3.3 times greater than in robot movements.

Conclusion. The results of the study suggest that the use of robotic laser systems in medicine, in particular the one developed by us for the maxillofacial region, can significantly improve the accuracy of medical laser movements, which is of prime importance in complex trajectories.

Study Funding. The study was supported by a grant as per the government task by the Ministry of Health of the Russian Federation 056-00139-16

Conflicts of Interest. The authors have no conflicts of interest related to the present study.

\section{References}

1. Chunikhin A.A., Bazikyan E.A., Krasnovsky A.A., Syrnikova N.V., Chobanyan A.G. The prospects for the improvement of the minimally invasive laser technologies for the photodynamic treatment of dental pathologies. Rossiyskaya stomatologiya 2015; 8(2): 71-74, https://doi. org/10.17116/rosstomat20158271-74.

2. Lehnert M.W. Lasers in medicine and dentistry. Northwest Dent 1996; 75(1): 17-22.

3. Goonewardene S.S., Persad R. Robotic radical prostatectomy, day-case surgery and cardiac health: an opposing paradigm? J Robot Surg 2015; 9(4): 355, https://doi. org/10.1007/s11701-015-0537-9.

4. Vasiliev A.O., Govorov A.V., Dyakov V.V., Rasner P.I., Kolontarev K.B., Maltsev E.G., Pushkar D.Yu. Modern possibilities of robotic technologies: experience of department of urology MSMSU. Farmateka 2016; S1: 44-47.

5. Robustova T.G., Bazikian E.A., Ushakov A.I., Daian A.V., Serova N.S., Ushakov A.A. Combined clinico-roentgological approach in reconstructive surgery and maxillary sinus lifting for dental implantation. Rossiyskaya stomatologiya 2008; 1: 61-68.

6. Chunikhin A.A., Sahakyan M.Y., Gazhva S.I., Bazikyan E.A. Development of nanosecond laser module built in the robotic multifunctional surgical complex for minimally invasive therapy of maxillofacial area pathology and estimation of its effects on blood plasma. Sovremennye tehnologii $v$ medicine 2016; 8(4): 30-35, https://doi.org/10.17691/ stm2016.8.4.04. 